\title{
Thermal and Optical Analyses of Retro-Reflector for Poloidal Polarimeter on ITER*)
}

\author{
Ryota IMAZAWA, Yasunori KAWANO, Takehiro ONO and Yoshinori KUSAMA \\ Japan Atomic Energy Agency, 801-1 Mukoyama, Naka 311-0193, Japan
}

(Received 6 December 2010 / Accepted 10 February 2011)

\begin{abstract}
This paper describes results from the first integrated analysis of the feasibility of a retro-reflector (RR) installed in the ITER vacuum vessel. We have carried out thermal and structural analyses of a passively-cooled $\mathrm{RR}$ and a terrace retro-reflector array (TERRA) using heat load conditions expected in ITER. In the case of the $\mathrm{RR}$, the maximum temperature is approximately $620^{\circ} \mathrm{C}$, and flatness of the deformed mirror surface of the $\mathrm{RR}$ is approximately $80 \mu \mathrm{m}$. Although maximal displacement of the mirror surface is large, $69 \%$ of the incident-laser power returns to the diagnostic room ( $89.9 \%$ returns when the RR is not deformed), phase disturbance is $\pi / 4$ and the rotation angle of the polarization plane is less than $0.06^{\circ}$. On the other hand, the maximum temperature of TERRA is approximately $350^{\circ} \mathrm{C}$, and flatness of the deformed mirror surface is less than $10 \mu \mathrm{m}$. The flatness of the mirror surface of a deformed TERRA is much smaller compared to the laser wavelength $(118 \mu \mathrm{m})$, but the global shape curves in a manner which disrupts the coherent phase of laser light. The curvature radius of TERRA is $21 \mathrm{~m}$, and $49 \%$ of the incident-laser power returns to the diagnostic room ( $88.6 \%$ returns when TERRA is flat), phase disturbance is $\pi / 5$ and the rotation angle of the polarization plane is less than $0.06^{\circ}$. Since the optimized incident angles of a flat and curved TERRA are different, real-time feedback control of the incident angle is necessary for applying TERRA to the ITER poloidal polarimeter.
\end{abstract}

(c) 2011 The Japan Society of Plasma Science and Nuclear Fusion Research

Keywords: retro-reflector, ITER, polarimeter

DOI: $10.1585 /$ pfr.6.2402032

\section{Introduction}

The poloidal polarimeter system will be installed in ITER to identify the safety factor profile, $q(r)$, in the plasma core region [1]. Far-infrared laser light, injected in a plasma, is reflected by a corner-cube retro-reflector (RR) and returns to a diagnostic room. The rotation angle of the polarization plane of the laser light provides information about the magnetic field and electron density of the plasma. The RR will be installed in the remote handling slots and/or in dedicated holes in a slot running poloidally in the center of the first wall panel of a blanket module [2]. Several studies on concept development [3], optimization of the viewing chord arrangement [4], neutron analysis [5,6], etc., have been done, but the effect of thermal distortion of the RR on optical propagation characteristics has not been investigated. This paper describes the results from an integrated analysis of the RR for the first time.

In our analyses, the feasibility of a conventional RR and a terrace retro-reflector array (TERRA) for ITER is investigated. TERRA is a type of RR array and has been proposed for the ITER poloidal polarimeter [7]. When the RR is used, the dedicated holes for the RR should be inclined in order for the incident light to reflect three times inside

author's e-mail: imazawa.ryota@jaea.go.jp

*) This article is based on the presentation at the 20th International Toki Conference (ITC20). the RR and travel back along the incident-light path. In addition, it is preferable that the incident light angle is perpendicular to the input aperture of the RR to achieve a large effective aperture [8]. However, it seems difficult to create such inclined holes due to the intricate system of cooling water pipes inside the first wall panels [9]. Advantages of TERRA include keeping the same effective aperture, reducing the size compared to a conventional RR, and simplifying design of the slot (and surrounding cooling pipes) (compare Figs. 1 (b) and (c)).

Although the RR and TERRA will be exposed to high neutron and radiation fluxes in ITER, they will be passively cooled by heat conduction to the first wall panels. In this paper, we discuss the feasibility of installing RR/TERRA in the first wall panel. We carry out thermal and structural analyses of RR/TERRA under ITER conditions using the commercial finite element code ANSYS, and discuss the effect on optical properties of thermal distortion of the RR and TERRA. Finally, we discuss the laser-incident angle as it affects the power of returning light.

\section{What is TERRA? [7]}

This section gives an overview of TERRA. A detailed discussion of TERRA was provided in [7]. As mentioned before, TERRA is an array of small, inclined RRs. In an optical sense, TERRA is equivalent to a Littrow grat- 


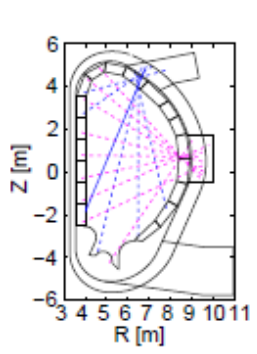

(a)

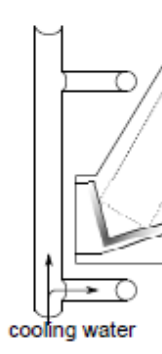

(b)

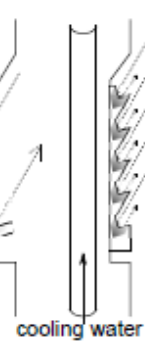

(c)

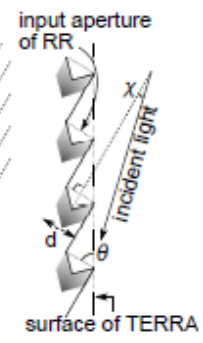

(d)
Fig. 1 (a) Viewing chords of the poloidal polarimeter in ITER. Blue and red lines show the viewing chords of the upper and equatorial port, respectively. The blue solid line is analysed in detail in this paper. Figures (b) and (c) illustrate cross sections of the RR buried in the wall at a tilt and TERRA, respectively. The figures include the water cooling pipes. The RR and TERRA are passively cooled by heat conduction to SS around the water cooling pipes. Figure (d) illustrates the definition of $d, \theta$ and $\chi$ used in Eq. (1).

ing, the blaze angle of which varies depending on the incident angle. Since light rays reflected by the different retroreflector cells have different phases, the reflected light interacts in a manner that generates bright and dark fringes. We have created the condition for the laser light reflected by TERRA to travel back along the same path as the incident laser light. The condition is expressed as:

$$
\frac{2 d(\tan \theta-\tan \chi) \cos \chi}{\lambda}=m,
$$

where $d$ is the interval of RR, $\theta$ is the angle between the normals of the RR and TERRA, $\chi$ is the angle between the normals of the RR and the incident light and $m$ is an integer (see Fig. 1 (d)). When TERRA is used, the laser-incident angle should always be controlled to satisfy this condition.

\section{Laser Transmission Optics}

In the following analysis, we focus on the viewing chord shown as the solid line in Fig. 1 (a). This chord is inclined at an angle of $67.19^{\circ}$ against the surface of the blanket module. We use the optical transmission parameters designed for this viewing chord [7]. The polarization of the laser is linear, and the wavelength is $118 \mu \mathrm{m}$. The laser travels from the diagnostic room $(z=0 \mathrm{~m})$, is reflected by 2 mirrors with a focal length of $15 \mathrm{~m}$ at $z=15 \mathrm{~m}$ and $z=45 \mathrm{~m}$, and reaches the RR at $z=60 \mathrm{~m}$. The position of the RR is the beam waist position, and the beam waist radius is $23 \mathrm{~mm}$.

\section{Integrated Analyses of the Conven- tional RR}

\subsection{Conditions for thermal and structural analyses}

We design the RR for the viewing chord as shown in Figs. 2 (a) and (b). The diameter of the input aperture of $\mathrm{RR}$ are $60 \mathrm{~mm}$ in order to be installed in the central slot
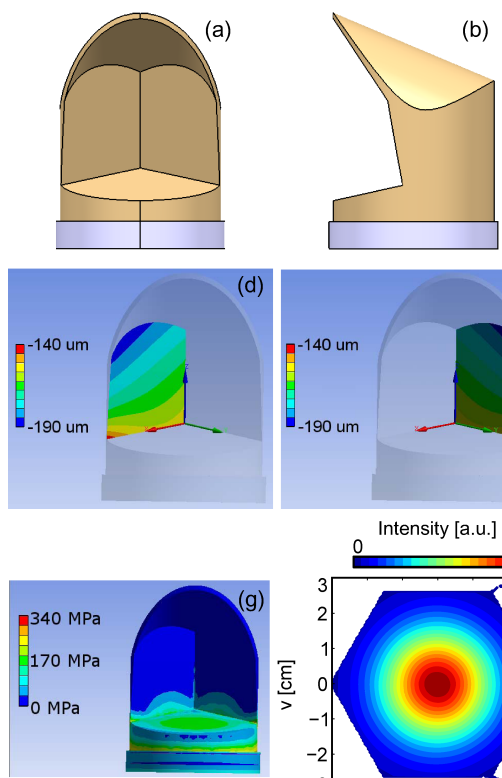

(b)
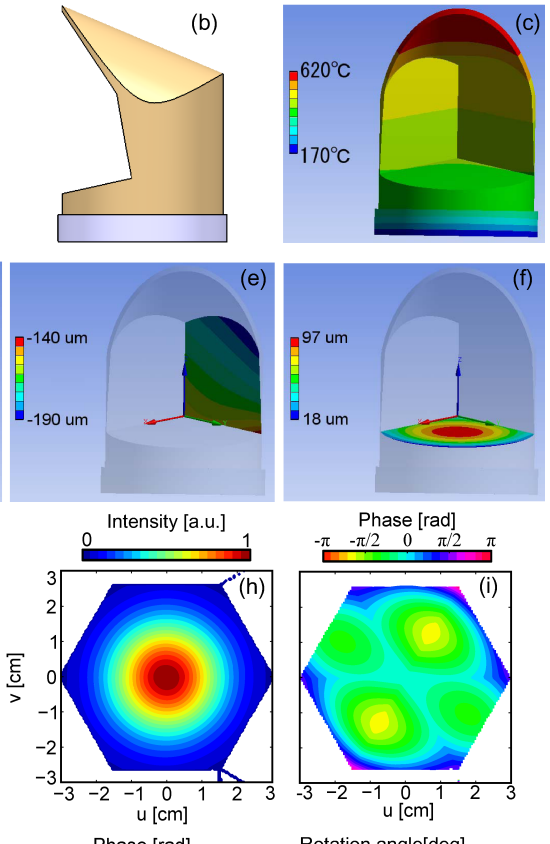

Phase [rad]
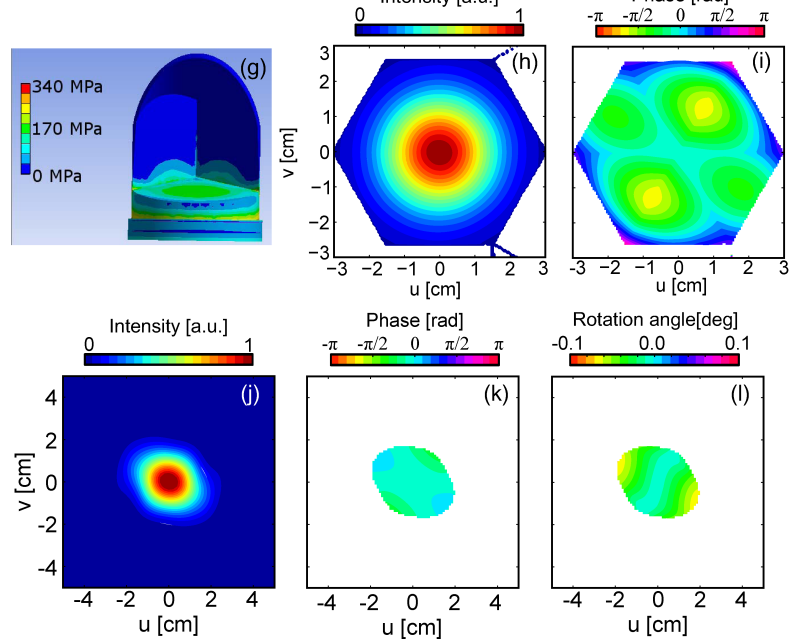

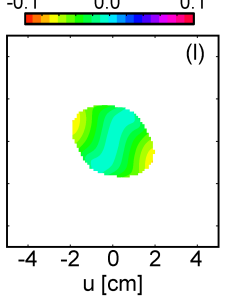

Fig. 2 Analysis model and results. Figures (a) and (b) illustrate the analysis model of the conventional RR and part of a blanket module. Figure (c) shows the temperature distribution, figures (d-f) show displacement normal to the mirror surface, and figure (g) shows the equivalent stress distribution. Figures (h) and (i) show the intensity and phase just after reflection, respectively. Figures (j), (k) and (l) show the intensity, phase and rotation angle of the polarization angle of light reflected by a deformed RR (i.e. at $z=0 \mathrm{~m}$ ), respectively. Plotted areas in figures (k) and (l) indicate where the intensity of light exceeds $I_{\max } / e^{2}\left(I_{\max }\right.$ : the maximal intensity).

running poloidally in the center of the first wall panel of the blanket module. Copper alloy $(\mathrm{CuCrZr})$ is the selected material for the RR because of its high thermal conductivity, high strength and radiation resistance. The RR has a visor to protect part of the mirror from plasma radiation. In this study, a simplified stainless-steel (SS) structure serves as the base of the RR instead of the specific design of a first wall panel, hence the RR is fastened to a SS plate whose thickness is $10 \mathrm{~mm}$. This SS plate corresponds to the part of the blanket module between the RR and a water cooling pipe (see Fig. 1). In our model, the surface of the SS plate is assumed to be cooled by water, except for the area touching the RR. The RR is cooled by heat conduction to the plate. The surface load from plasma irradiation is $0.15 \mathrm{MW} / \mathrm{m}^{2}$, and the body load from nuclear heating is $5 \mathrm{MW} / \mathrm{m}^{3}$ [10]. The heat transfer coefficient of the watercooled surface is $10 \mathrm{~kW} / \mathrm{m}^{2} \mathrm{~K}[10,11]$, and the temperature 
of the water is $140^{\circ} \mathrm{C}$.

\subsection{Results of conventional RR}

Figs. 2 (c-g) show results of thermal and structural analyses of the RR. Although the RR and the SS plate are ideally connected in our model, the temperature of the RR exceeds $600^{\circ} \mathrm{C}$. That is to say, a passively-cooled RR with a diameter of $60 \mathrm{~mm}$ is not sufficiently cooled regardless of the method of attachment (e.g. bolting, brazing, welding), and a dedicated cooling water pipe is absolutely necessary if a RR equivalent in size to the central slot is installed in the blanket module. The yield strength at $0.2 \%$ offset, $\sigma$, for $\mathrm{CuCrZr}$ is given by [12]:

$$
\sigma=-4 \times 10^{-4} T^{2}-5 \times 10^{-2} T+3 \times 10^{2},
$$

where $T$ is temperature in Celsius and $\sigma$ is in MPa. The stress placed upon our design barely satisfies this criterion. The stress on the mirror surface closest to the SS plate is higher than that placed on the other surfaces and equals $180 \mathrm{MPa}$, which is smaller than the yield strength for the temperature of the mirror surface $\left(400^{\circ} \mathrm{C}\right)$. The mirror surface closest to the SS plate is the most deformed. As shown in Fig. 2 (f), the flat mirror deforms into a convex mirror. Flatness of the deformed surface is approximately $80 \mu \mathrm{m}$. Displacement of the other two mirror surfaces shown in Figs. 2 (d) and (e) is larger than that of the mirror closest to the SS plate, but flatness is largely conserved.

Using these deformed mirror shapes, we calculated the propagation of the laser light reflected by the RR. Figs. 2 (j), (k) and (1) show the intensity, phase and rotation angle of the polarization angle of light reflected by the deformed $\mathrm{RR}$ at $z=0 \mathrm{~m}$ (the diagnostic room), respectively. Assuming that the mirror surfaces exhibit perfect reflection, $68.9 \%$ light power of the incident laser returns to the diagnostic room, polarization plane is altered by $0.06^{\circ}$, and the phase disturbance is $\pi / 4$. On the other hand, when the RR is not deformed, $89.9 \%$ light power of the incident laser returns, the angle of the polarization plane is conserved and the phase distribution is uniform. This value, $89.9 \%$, is due to the fact that the radius of the RR $(30 \mathrm{~mm})$ in our design [7] was limited by the design of the blanket module and was not sufficiently larger than the beam radius at the RR (23 mm). Light power returning to the diagnostic room decreases by $23 \%$ because of deformation.

\section{Integrated Analyses of TERRA \\ 5.1 Conditions for thermal and structural analyses}

We use the same viewing chord as in the earlier sections to carry out thermal, structural and optical analyses of TERRA as illustrated in Figs. 3 (a-c). TERRA has a honeycomb structure, and the input aperture of each RR forming TERRA is a regular hexagon. The radius of the input aperture of a RR cell is $3 \mathrm{~mm}$ [7]. The thickness of TERRA is $7 \mathrm{~mm}$ and is 10 times smaller than the height of the RR $(78 \mathrm{~mm})$ due to the small input aperture of the RR cell. When TERRA is viewed from the direction of incident light, the shape of TERRA is a regular hexagon (Fig. 3 (c)), and the diameter of the circumscribed circle of the hexagon is $60 \mathrm{~mm}$ (same as the RR input aperture). The material of TERRA is $\mathrm{Cu}$-alloy $(\mathrm{CuCrZr})$.

In this model, TERRA is attached to a hollow rectangular SS pipe, whose interior dimensions (water area) at the top are $160 \times 35 \mathrm{~mm}$ [11]. Heat load conditions for TERRA are exactly the same as those for the RR. That is to say, the heat transfer coefficient of the rectangular pipe is $10 \mathrm{~kW} / \mathrm{m}^{2}[10,11]$, and the thickness of the wall between TERRA and water is $10 \mathrm{~mm}$.

\subsection{Results of thermal and structural analy- ses}

Figs. 3 (d) and (e) show the results of the thermal analysis. The maximal temperature of TERRA is approximately $350^{\circ} \mathrm{C}$. The yield strength at $0.2 \%$ offset for $\mathrm{Cu}-$ $\mathrm{CrZn}$ is approximately $230 \mathrm{MPa}$ at $350^{\circ} \mathrm{C}$, and the stress placed on our design exceeds this criteria (see the red area in Fig. $3(\mathrm{e}))$. Therefore, further improvements in the mechanical design (e.g. slit, chamfer) are necessary. Flatness of the deformed mirror surface is less than $10 \mu \mathrm{m}$. Although the flatness of deformed mirror surface is negligible compared to the laser-wavelength, the global shape of TERRA is curved. The curvature radius of TERRA obtained by structural analysis is $21 \mathrm{~m}$.

\subsection{Optical analysis of light reflected by a curved TERRA}

When TERRA is curved, the RR cells do not align uniformly, the input apertures of the RRs are not parallel to each other, and the three mirrors in each RR cell are not mutually perpendicular. We have developed a method to calculate the propagation of light reflected by a curved TERRA.

First, we explain the model of the curved TERRA configuration. Let $\left(x_{\mathrm{V}}, y_{\mathrm{V}}, z_{\mathrm{V}}\right)$ be the coordinates of vertex of the RRs of a flat TERRA. The surface of TERRA is on a plane $z=0 \mathrm{~m}$, and all vertices are in the region of $z \leq 0$. The curvature center is expressed as $(0,0,-R)$, where $R(>0)$ denotes the curvature radius. The vertex of the curved TERRA is defined as:

$$
\begin{aligned}
& x_{\mathrm{V}}^{\prime}=R^{\prime} \sin \phi \cos \theta, \\
& y_{\mathrm{V}}^{\prime}=R^{\prime} \sin \phi \sin \theta, \\
& z_{\mathrm{V}}^{\prime}=R^{\prime} \cos \phi-R^{\prime},
\end{aligned}
$$

where $R^{\prime}=R+z, \phi=\sqrt{f_{x}^{2} x^{2}+f_{y}^{2} y^{2}} / R^{\prime}, \theta=$ $\tan ^{-1}\left\{\left(f_{y} y\right) /\left(f_{x} x\right)\right\}$, and $f_{x} / f_{y}$ denotes the extension ratio of the TERRA surface in the $x / y$ direction. The positions of vertices are deformed, and the mirror surfaces are assumed to be flat.

We posit an initial plane, which is perpendicular to the incident laser light and is as close as possible to a curved 


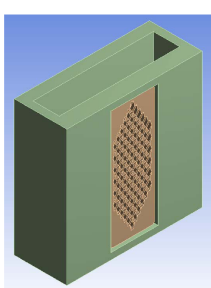

(a)

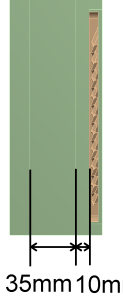

(b)

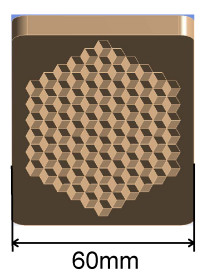

(c)

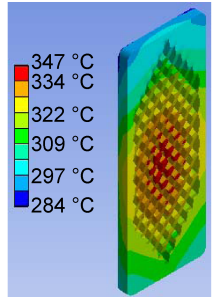

(d)

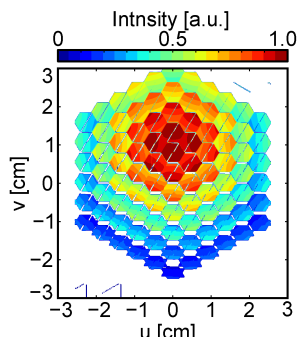

(f)

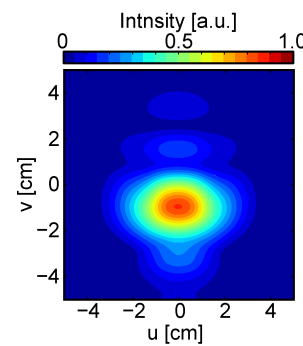

(h)

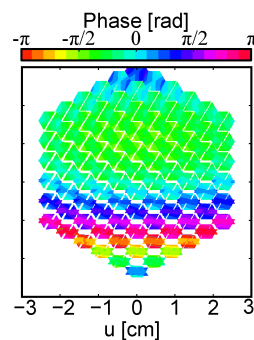

(g)

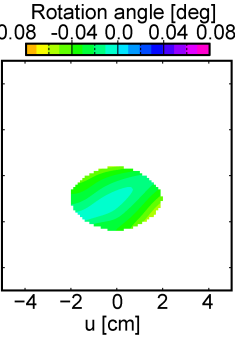

(j)
Fig. 3 Analysis model and results. Figures (a-c) illustrate the analysis model of TERRA and part of a blanket module. Figures (d) and (e) show temperature and the equivalent stress distribution, respectively. Figures (f) and (g) show the intensity and phase of light just after reflection. Figures (h), (i) and (j) show the intensity, phase and rotation angle of the polarization angle of light reflected by a curved TERRA at $z=0 \mathrm{~m}$ (the diagnostic room), respectively. Plotted areas in figures (i) and (j) show where the intensity of light exceeds $I_{\max } / e^{2}\left(I_{\max }\right.$ : the maximal intensity).

TERRA. Methods of optical analyses are different in 2 regions; (i) ray-tracing between the initial plane and TERRA and (ii) diffraction between the initial plane and the diagnostic room. In area (i), light returns the initial plane to TERRA, is reflected by TERRA and returns to the initial plane. We calculated the phase and polarization angle of light returning to the initial plane by using the ray-tracing method. All mirror surfaces are assumed to have perfect reflectivity, and the electromagnetic field, $\vec{E}$, of the reflected light is given by $\vec{E}_{\mathrm{r}}=-\left(\vec{E}_{\mathrm{i}} \cdot \vec{s}_{\mathrm{i}}\right) \vec{s}_{\mathrm{r}}+\left(\vec{E}_{\mathrm{i}} \cdot \vec{p}_{\mathrm{i}}\right) \vec{p}_{\mathrm{r}}$, where $\vec{s} / \vec{p}$ denotes the direction of s-/p-polarization and i/r denotes incident/reflected light. Next, in area (ii), we calculate the propagation of light from the initial plane to the diagnostic room by using the Fresnel-Kirchhoff diffraction formula.

\subsection{Results for a curved TERRA}

Figs. 3 (f) and (g) show the intensity and phase of light

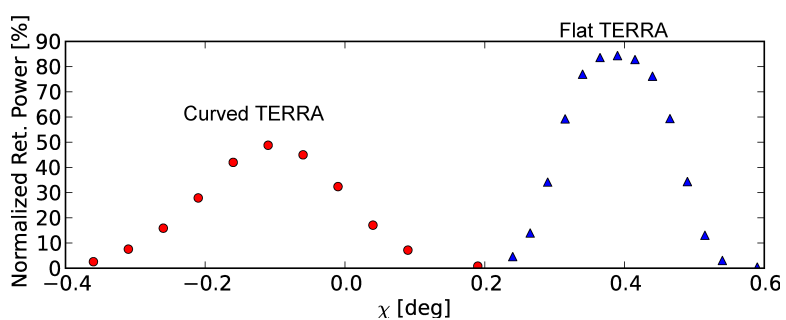

Fig. 4 The ratio of returning power to the initial power as a function of the incident angle $\chi$. The symbols "o" and " $\triangle$ " denote a curved TERRA with a radius of $21 \mathrm{~m}$ and a flat TERRA, respectively.

just after reflection. When TERRA is used, the incident angle should be controlled to satisfy Eq. (1), i.e., for unifying the phase of reflected light. However, the area of a uniform phase is limited on a curved TERRA. Figs. 3 (h), (i) and (j) show the intensity, phase and rotation angle of the polarization angle at $z=60 \mathrm{~m}$, respectively. The light power returning to the diagnostic room is $49 \%$ of the light power sent initially to TERRA, the phase disturbance is $\pi / 5$ and the rotation angle of the polarization plane is less than $0.06^{\circ}$. On the other hand, when TERRA is flat, $88.6 \%$ light power of the incident laser returns, the angle of the polarization plane is conserved and the phase distribution is uniform. Light power returning to the diagnostic room decreases by $45 \%$ because of deformation.

Fig. 4 shows the ratio of returning power to initial power as a function of the incident angle $\chi$ for both a flat TERRA and a curved TERRA with a radius of $21 \mathrm{~m}$. When TERRA is flat, the maximum returning power is obtained at $\chi=0.39^{\circ}$, which satisfies Eq. (1). On the other hand, when TERRA is curved with a radius of $21 \mathrm{~m}$, the maximum returning power is obtained at $\chi=-0.11^{\circ}$. Therefore, the optimized incident angle would be expected to change during a single plasma operation, and real-time feedback control of the incident angle will be necessary.

\section{Discussion}

As described in this paper, we have obtained the intensity, phase and polarization angle of light reflected by a deformed RR and TERRA to the diagnostic room. However, to confirm the feasibility of RR/TERRA under severe ITER load conditions, further investigation is needed. In order to clarify feasibility, we need to evaluate the fabrication accuracy of TERRA, the feedback control scheme for the laser-incident angle and the characteristics of other equipments of the polarimeter system, e.g., reflectivity degradation caused by deposition of impurities, change in laser power/quality, limited detector sensitivity, etc.

\section{Conclusions}

We have carried out an integrated analysis of a passively-cooled RR and TERRA for the first time using heat load conditions for ITER $\left(0.15 \mathrm{MW} / \mathrm{m}^{3}\right.$ surface load 
from plasma irradiation and $5 \mathrm{MW} / \mathrm{m}^{3}$ body load from nuclear heating load). A simplified SS structure serves as the base of the RR and TERRA instead of a specific design of a first wall panel, and the RR and TERRA are installed at a distance of $10 \mathrm{~mm}$ from a water cooling pipe.

In the case of the RR, the maximal temperature exceeds $600^{\circ} \mathrm{C}$, the stress barely satisfies the yield strength at $0.2 \%$ offset, and the flatness of the deformed mirror surface is approximately $80 \mu \mathrm{m}$. Although the distortion is equivalent to the laser-wavelength $(118 \mu \mathrm{m}), 68.9 \%$ of the initial laser power returns to the diagnostic room, phase disturbance is $\pi / 4$ and the rotation angle of the polarization plane is less than $0.06^{\circ}$. Light power returning to the diagnostic room decreases by $23 \%$ because of deformation.

In the case of TERRA, the maximal temperature of TERRA is approximately $350^{\circ} \mathrm{C}$, and the flatness of the deformed mirror surface is less than $10 \mu \mathrm{m}$. The flatness of the mirror surface in the deformed TERRA is much smaller than the laser wavelength $(118 \mu \mathrm{m})$, but the global shape is curved with a radius of $21 \mathrm{~m}$. When the light is reflected by TERRA with a $21 \mathrm{~m}$ curvature radius, $49 \%$ of the initial power returns to the diagnostic room, phase disturbance is $\pi / 5$ and the rotation angle of the polarization plane is less than $0.06^{\circ}$. Light power returning to the diagnostic room decreases by $45 \%$ because of deformation. When TERRA is curved, Eq. (1) does not hold. In this case, the optimized incident angles for a flat and curved TERRA are different.
Our optical analysis suggests that real-time feedback control of the incident angle is required.

\section{Acknowledgments}

The views and opinions expressed herein do not necessarily reflect those of the ITER Organization.

[1] A. E. Costley et al., Proceedings of the 22nd IAEA Fusion Energy Conference, Geneva, Switzerland, 13-18 Octorber 2008 (IAEA, Vienna, 2009), Paper No. IT/P6-21.

[2] M. Merola et al., Fusion Eng. Des. 85, 2312 (2010).

[3] A. J. H. Donné et al., Rev. Sci. Instrum. 75, 4694 (2004).

[4] T. Yamaguchi et al., Plasma Phys. Control. Fusion 50, 045004 (2008).

[5] M. Ishikawa et al., J. Plasma Fusion Res. SERIES 9, 43 (2010).

[6] M. Ishikawa et al., "Neutronic Analysis of the ITER Poloidal Polarimeter," to be published in Fusion Eng. Des. Special Issue (2011).

[7] R. Imazawa et al., Rev. Sci. Instrum. 82, 023116 (2011).

[8] D. D. A. Arnold, Tech. Rep. (NASA Grant NGR 09-015196; NASA Cr-130696, 1972).

[9] Y. Kawano et al., The 8th Joint Conference for Fusion Energy (10-11 June 2010, Takayama, Japan) 10B-48P.

[10] K. Weishan et al., Plasma Sci. Technol. 12, 628 (2010).

[11] F. Zhang et al., 21st IAEA Fusion Energy Conference, Chengdu(China), IT/PT2-2 (2006).

[12] S. J. Zinkle, DOE/ER-0313/28, p.171-175 (2000); S. J. Zinkle, DOE/ER-0313/21, p.165-174 (1996). 